\title{
Using Telehealth to Implement Intervention Services with Young Children
}

\author{
Ruby Natale, Kristyna Pena, Alexandra Blanco, Ellen Kolomeyer, Julie D’Amico, and Emperatriz \\ Guzman
}

\begin{abstract}
Intervention services positively impact outcomes of children with special needs and their families. However, families face many burdens when accessing high-quality intervention services such as availability of providers, time, and cost; these burdens are often magnified for underserved families. As such, there is a high need to determine if providing services via telehealth is acceptable to reaching these families. To fill this gap in the field, we developed "Early Discovery" an telehealth prevention education program tailored specifically for high risk preschool age children. This framework aided in identifying successful intervention components that can be widely disseminated with the ultimate goal of serving families that can be aided via telehealth.
\end{abstract}

Index Terms - Telehealth, preschool children, intervention.

\section{INTRODUCTION}

The goal of early intervention services is to improve outcomes for infants and toddlers with developmental delays as well as to provide service coordination and appropriate supports for these children's families. Research on early intervention for children with developmental delays suggests that early identification of a developmental delay or disability is associated with more positive outcomes of interventions designed to remediate the delay [1]. Additionally, it has been argued that children with delays who receive early intervention services are more likely to be ready to learn when they enter school [2].

Delgado, Vagi, and Scott [3] examined outcomes for over 2000 preschool children identified with developmental delays in the state of Florida and found that $75 \%$ of these children were receiving special education services in third grade. Of these, $34 \%$ were identified as having a specific learning disability; $20.5 \%$, a mild intellectual disability; $10.3 \%$, specific language impairment; $9.1 \%$, emotional disturbance; $7.37 \%$, speech impairment; and $6.3 \%$, a diagnosis of autism. Of those children not receiving special education services, $15 \%$ had been retained in either first or second grade, a rate that is substantially higher than that for the general regular education population. Findings of this study suggest that the majority of children with identified delays in preschool continue to require special services once they enter school.

Disruptive behavior problems in early childhood constitute a significant problem for children, their parents, and society.

Manuscript received January 9, 2019; revised April 14, 2019. This work was supported by the Children's Trust of Miami-Dade county

The authors are with the University of Miami, Miami, FL, 33136, USA (e-mail: rnatale@med.miami.edu, KPena@med.miami.edu, ab@med.miami.edu,Ek@med.miami.edu, eguzman@med.miami.edu).
Prevalence estimates of disruptive behavior disorders in preschool children are over 10\% [4]. Research demonstrated a pattern of long-term chronicity for a subset of children who exhibit early disruptive behavior [5]. Early intervention is helpful in reducing the chronicity of disruptive behavior problems [6].

Many families are unable to engage in interventions due to a variety of barriers such as high costs, difficulty scheduling, transportation problems, etc. [7]. These barriers particularly affect lower income families, as reflected by the increased rates of treatment dropout associated with low SES [8]. Given that low-SES families are often at risk of experiencing higher levels of stressors and subsequent mental health problems, it is imperative that evidence-based treatments be modified to increase accessibility for these families.

In order to make services more accessible and to reduce barriers, telehealth can be an option. Research has shown benefits that include being more cost-effective [9]. For example, it has been found that $87 \%$ more children could have access to therapy services by using telehealth without increasing the costs [10]. Another study showed that professional costs are much higher for in-home therapy models vs. telehealth models. One study of 107 children with developmental disability [11] looked at those that received telehealth services compared to those with in-home sessions. Results showed that telehealth conditions provided a more efficient usage of professional paid time. Using telehealth can also result in reducing the number of miles that families need to travel in order to receive services and thus saves money for the families as well as professional costs. One study found that families can spend about 2,263-miles to travel to therapy and telehealth would eliminate this need [12].

\section{Procedures}

\section{A. Procedure}

Data was analyzed for sixteen families who participated in the Early Discovery program. Once there was commitment and a signed consent form from parents, Early Discovery staff met with parents to establish an individual plan that details intervention services. A hybrid telehealth intervention group consisted of children and their families who received Early Discovery services to address behavior or speech or developmental concerns via a combination or in-home sessions and telehealth sessions. Pre and post assessment was conducted in person at the beginning and end of the intervention.

\section{B. Intervention}


Early Discovery (ED) is a comprehensive early childhood prevention program designed to support families and mildly delayed young children who do not meet eligibility for Part B and $C$ services but who needed intervention and resources. The goals of ED are to: 1) provide children with high-quality services; 2) support, educate and empower parents/caregivers to maintain children's progress; 3 ) link families to available community resources; and 4) reduce the need for special education services later on in life. Services include speech/language, occupational, behavioral, and/or general developmental intervention. It is our intention to go above and beyond to ensure that children are receiving the best care, all children are able to access services, and that we are conducting evidenced-based work in collaboration with the community.

All families received the same number of sessions total sessions (12 sessions). The Children's Trust of Miami-Dade County funded Early Discovery to provide early intervention services to children birth through 5 years with mild developmental delays.

Referral Stage: To be referred to Early Discovery, children must meet the following criteria: 1) be 0-5 years-old; 2) have an evaluation indicating 10 to $29 \%$ delay (i.e. standard score 71-84) in any area of a standardized assessment; 3) are ineligible for services through Part B/C of IDEA.; 4) services are not covered by private insurance or Medicaid; and 5) services are not currently being received elsewhere.

Care Coordination Stage: All qualifying referrals are assigned to a coordinator for a face-to-face-intake meeting at the most convenient location for the parent (home, school, work, etc.). The caregiver completes enrollment forms and a Strengths, Weaknesses, Opportunities, and Threats (SWOT) analysis, a method used to assist with planning goals and setting priorities (see attachment in Continuous Learning \& Quality Supports) to capitalize on the family's strengths and address needs in order to connect them to community resources.

With each case, our care coordinators s are responsible for the following:

1) Conduct an Intake utilizing motivational interviewing, a strengths-based approach to elicit positive changes in behavior. Intakes are face-to-face in the location most convenient for the family. They will perform a SWOT analysis that assesses the family's needs, priorities, and strengths to create a Family Action Plan, and determines the fit with telehealth. They will identify any insurance coverage and also verify income as per our sliding scale for any applicable fees.

2) Within 72 hours of meeting, they review the intake information, provide resources, and assign the appropriate intervention/therapy and service provider.

3) Manage child's services by referring the families to the resources in their CoP through a warm-hand-off by giving them the name and phone number of the identified contact person at the partner agency. They will follow-up to ensure contact was made by communicating with the caregiver at least 2 times a month either in person, phone or via telehealth. Follow-up sessions will ensure caregiver/child intervention sessions are meeting the families' unique individual needs. They will shadow sessions, continuously assess child and families' additional needs to link to community resources, and monitor child's developmental progress and parental satisfaction.

4) Meet with the family to complete a parent satisfaction survey and discharge from the program; review post-evaluation outcomes and developmental progress; and identify continued needs to facilitate transition of care into another program (e.g. Advocacy Network).

Treatment Stage: Licensed Graduate Level Therapists conduct baseline/pre-testing to ensure eligibility requirements of a $10-29 \%$ delay and for treatment planning. The first session consists of informing the parent of the test results and working together to form an Individual Service Plan tailored to the specific needs of the child.

A toolkit and consistent protocol is followed for treatment for each service. We offer flexible hours from $7 \mathrm{am}$ to $7 \mathrm{pm}$, weekends, and convenient locations of services including parent homes, childcare centers, and offices of our partner agencies.

\section{Implementation:}

1) Pre-Evaluation is conducted using the PLS-5 and observing 10 minutes of a Play-Based Assessment to assess parent-child interaction.

2) Session 1: Family is given strengths-based feedback based on the evaluation results. Caregiver and therapist work together to develop goals to add to the Family Action Plan originally developed with the Care Coordinator. This approach ensures engagement - adapting services to child's needs and family's cultural/personal priorities; identifying the child's interests (e.g. toys, games, books) to facilitate engagement; and building on the strengths.

3) Session 2- Therapist builds rapport and conducts an in-person telehealth tech assistance session with families prior to services.

4) Sessions 3 to 12 (or up to 20): 1-3 times per week for up to 10-20 sessions by implementing evidence-based Toolkit. Lesson plans incorporating parent-/teacher-coaching/child-directed activities are consistent with our current Short Term Intensive Intervention model.

5) Hybrid telehealth model- 4 sessions are delivered in-person and 4 via telehealth (but this can be adjusted based on families individual needs). Telehealth is meant to facilitate parental involvement in language-rich situations. A carry-over goal will be provided at the end of each session to implement in high-frequency at home during daily activities. If needed, co-intervention can be utilized in the form of 6 consultations with another professional about the child's additional non-speech and language related concerns. This facilitates global developmental progress. (*See telehealth activity for further details and barriers/solutions)

6) Post-Evaluation/Transition Planning is conducted using the PLS-5 to determine developmental progress and continued needs. The interventionist/therapist will meet with caregiver to review the child's developmental outcomes. The Care Coordinator will help the family 
transition into another program (e.g. Advocacy Network, Florida Alliance for Assistive Services and Technology etc.) should there be continued service needs.

\section{Engagement/Tailoring:}

All therapies follow the same engagement/tailoring plan.

1) Care Coordinators do bi-weekly follow-ups, interventionists follow-up with families after each session and strengths-based feedback is provided throughout service implementation to build parent efficacy. Services are adapted to the needs of the family based on assessment and caregiver culture, education, literacy, and skill.

2) A Family Action Plan is developed for each child to ensure that the intervention is followed through outside of teaching sessions in order to best promote development and social-emotional functioning.

3) Services are tailored so that the same intervention can be used with all caregivers in the child's life including teachers, parents, grandparents, etc., allowing for a continuity of care approach.

4) Families are served at the time and location most convenient to them and by service providers who speak their same language. Telehealth contingency plans have been developed so that all are "ED Connected" (access either by borrowing telehealth equipment, using mobile phones, or accessing hubs that have been identified throughout the county).

5) All service providers use motivational interviewing skills, psychoeducation, and parent-coaching to cope with treatment resistance, address continued service needs, and facilitate transition of care.

A system is in place to establish clear communication between the provider and the Family Care coordinator. It is ideal that a discharge meeting occur with the provider/therapist, parent and/or teacher (if applicable), and family care coordinator present. It is essential that the parent understands what they need to do to maintain and continue the child progress on a positive developmental path; therefore, the discharge meeting provides parents with clear recommendations. In addition, the therapist communicates with the parent (and teacher when possible) on a weekly to provide consistent feedback to the family.

Completion of the Weekly Progress Note allows us to track the progress of the children enrolled in Early Discovery. The progress notes are family friendly and are written in layman's terms. There are sections in which to communicate activities that teachers and parents can engage in to help children practice and maintain their new skills. The progress note is a session summary that is helpful to the caregiver/teacher and parent. If the family speaks Spanish, the note is written in Spanish so they are able to read what is occurring in session. Depending on the level of improvement shown, a child may be discharged from services or referred to Early Steps or FDLRS Child Find for further evaluation.

\section{Measurement Tools}

Preschool Language Scales, Fifth Edition (PLS-5). The PLS-5 [13] was individually administered and assessed receptive and expressive language. Administration time ranged from 45 to 60 minutes. Subscales on the PLS-5 evaluated receptive and expressive language skills in the areas of attention, play, gesture, vocal development, social communication, semantics, language structure, integrative language skills, and emergent literacy skills. Both receptive and expressive domains were administered and the standard scores were calculated for each domain (typical population: $M=100, S D=15)$. Standard scores between 85 and 115 indicated average functioning. Standard scores between 71 and 84 indicated a mild delay in language development and qualified children to participate in Early Discovery.

The Devereux Childhood Assessment (DECA). The DECA [14] is a 37-item behavior rating scale which measured protective factors in children ages 2 through 5 years old. Parents rated 27 positive behaviors that comprised a Total Protective Factors composite and 10 behavioral concerns exhibited by preschoolers on a 5-point Likert scale of frequency ranging from 0 "never" to 4 "very frequently." The DECA provided scores in the following domains: a) Initiative, b) Self-control, and c) Attachment, which comprise the Total Protective Factors composite, and d) Behavioral Concerns. Higher scores were optimal on the Initiative, Self-control, and Attachment subscales, whereas a lower score was more optimal on the Behavioral Concerns subscale. For the purposes of this study, the Initiative, Self-control, Attachment, and Behavioral Concerns subscales were examined

\section{RESULTS}

\section{A. Population}

Sixteen children ages 1 year to 4 years of age participated in the program from Miami-Dade County. Three were female and thirteen were male. $81 \%$ were Hispanic and $18.8 \%$ were Non-Hispanic. $68 \%$ of the families were married. $43 \%$ had less than a college degree.

\section{B. Speech Therapy Outcomes}

The PLS-5 pre- and post-intervention services was administered to those with speech delays. Consistent with programmatic goals, analyses showed significant improvements in expressive domains. The expressive language mean standard score $(M=73 S D=9.5)$ fell within the mildly delayed range pre-intervention and improved to the average range $(M=94.3, S D=6 / 65)$ post-intervention, $t(2)=$ $-3.55, p<.07)$ for the telehealth group. For the in home group, the expressive language mean standard score $(M=78.33$ $S D=3.2$ ) at pre-intervention increased to 92.00, $S D$ 6.08); $t(2)=p<.09$ ) however, the improvement is noteworthy nonetheless, as ED aims for global improvement in addition to the treatment target.

\section{Behavior Therapy Outcomes}

Children who received behavioral therapy were administered the DECA pre- and post-intervention. Results showed significant improvement across all domains. Mean T-scores improved on Behavioral Concerns pre- $(M=69.75$, $S D=3.03)$ to post-intervention $(M=54, S D=3.55), t=8.15$, $p<.004$ for the telehealth group. Mean T-scores improved on Behavioral Concerns pre- $(M=58.75, \quad S D=15.34)$ to post-intervention $(M=44.50, S D=10.87), t=5.71, p<.011$ for 
the in-home group.

\section{DISCUSSION}

As projected, children who participated in speech/language therapy and behavior therapy showed clinically significant improvements in each respective developmental domain from pre- to post-treatment regardless if they received telehealth or in-home sessions. At the end of therapy, scores were comparable to typically developing children on language indices and behavioral concerns. These outcomes were similar to findings of short-term intensive early intervention services that served children with moderate to severe disabilities [14]-[16] and revealed positive impacts of Early Discovery.

In addition, the children in both groups (telehealth vs. traditional intervention) showed statistically significant increases in development. This finding is corroborated by other research studies. For example, one study found increases in child social-communicative behavior [17] with no differences between telehealth and in-home service models. Another study found that telehealth and traditional models were both effective in decreasing problem behavior in children with Autism Spectrum Disorder [18].

Given that telehealth is more cost-effective, yet childhood outcomes are just as good when compared to traditional models, leads researchers to speculate that future research should determine a model for telehealth that can be used across settings.

Furthermore, it is recommended that when replicating this program to note several areas of success. For example, we learned that it is important for services to take place in the natural environment: either in the child's home, child care center classroom or as a last resort in the provider's office. Priority should be placed on meeting the needs of the family and providing the services at a location that best meets the family's needs. Early Discovery's mission is to build a system of care that will reduce the number of young children needing special education services by the time they begin kindergarten. Our mission is to help the children reach their developmental milestones, prevent the progression of any current delays, and reduce the risk of developing more severe delays in the future.

\section{CONCLUSION}

Children with identified delays or special needs in early childhood who receive appropriate, responsive early intervention are more likely to develop to their full potential. The longer concerns remain undetected or untreated, the more likely affected children will experience long-term challenges that can become increasingly more costly, complicated and less responsive to intervention. Early Discovery as a telehealth program can act as a model for other clinical and research programs targeting at-risk children to prevent declines in abilities that may otherwise require intense and costly exceptional student education. More research and cost benefit analyses of telehealth service would lead to improvements in policies and intervention services.

\section{ACKNOWLEDGMENT}

The authors would like to thank the families, children, and childcare providers that participated in this program and The Children's Trust of Miami-Dade County.

\section{REFERENCES}

[1] M. J. Guralnick, "Early intervention for children with intellectual disabilities: Current knowledge and future prospects," Journal of Applied Research in Intellectual Disabilities, vol. 18, pp. 313-324, 2005.

[2] Council on Social Work Education. (2015). 2015 Educational Policy. [Online]. Available: http://www.cswe.org/File.aspx?id=72120

[3] C. E. F. Delgado, S. J. Vagi, and K. G. Scott, "Tracking preschool children with developmental delay: Third grade outcomes," American Journal on Mental Retardation, vol. 111, no. 4, pp. 99-306, 2006.

[4] J. V. Lavigne et al., "The prevalence of ADHD, ODD, depression, and anxiety in a community sample of 4-year-olds," Journal of Clinical Child and Adolescent Psychology, vol. 38, no. 3, pp. 315-328, 2009.

[5] C. J. Trentacosta et al., "Longitudinal prediction of disruptive behavior disorders in adolescent males from multiple risk domains," Child Psychiatry and Human Development, vol. 44, no. 4, pp. 561-572, 2013.

[6] P. Fonagy, "Prevention, the appropriate target of infant psychotherapy," Infant Mental Health Journal, vol. 19, no. 2, 1998.

[7] M. E. Harrison, M. M. McKay, and W. M. Bannon, "Inner-city child mental health service use: the real question is why youth and families do not use services," Community Ment Health J., vol. 40, no. 2, pp. 119-131, 2004.

[8] M. A. Fernandez et al., "Treatment outcome for low socioeconomic status African American families in parent-child interaction therapy: A pilot study," Child \& Family Behavior Therapy, vol. 33, no. 1, pp. 32-48, 2011.

[9] D. F. Gros et al., "Delivery of evidence-based psychotherapy via video telehealth," Journal of Psychopathology Behavior Assessment, vol. 35, pp. 506-512, 2013.

[10] J. Cason et al., "Overview of states' use of telehealth for the delivery of early intervention (IDEA Part C) services," International Journal of Telerehabilitation, vol. 4, no. 2, pp. 39-46, 2012.

[11] S. Lindgren et al., "Telehealth and autism: Treating challenging behavior at lower cost," Pediatrics, vol. 137, no. S2, p. e20152851O, 2016.

[12] L. Heitzman-Powell et al., "Formative evaluation of an ABA outreach training program for parents of children with autism in remote areas," Focus on Autism and Other Developmental Disabilities, vol. 29, no. 1, pp. 23-38, 2013.

[13] I. L. Zimmerman et al., "PLS-5: Preschool language scale-5 [measurement instrument]," San Antonio, TX: Psychological Corporation, 2011.

[14] S. Eldivik et al., "Meta-analysis of early intensive behavioral intervention for children with autism," Journal of Clinical Child \& Adolescent Psychology, vol. 38, no. 3, pp. 439-450, 2009.

[15] P. A. LeBuffe and J. A. Naglieri, "The devereux early childhood assessment," Lewisville, NC: Kaplan Press Publishing, 1999.

[16] M. J. Guralnick, "Early intervention for children with intellectual disabilities: An update," Journal of Applied Research in Intellectual Disabilities, vol. 30, no. 2, pp. 211-229, 2017.

[17] T. Smith et al., "Randomized trial of intensive early intervention for children with pervasive developmental disorder," American Journal on Mental Retardation, vol. 105, no. 4, pp. 269-285, 2000.

[18] G. S. Vismara et al. "Telehealth for expanding the reach of early autism training to parents," Autism Research and Treatment, 2012, Article ID 121878 .

Copyright (C) 2019 by the authors. This is an open access article distributed under the Creative Commons Attribution License which permits unrestricted use, distribution, and reproduction in any medium, provided the original work is properly cited (CC BY 4.0).

Ruby Natale is an associate professor of clinical pediatrics at the University of Miami Miller School of Medicine in the Department of Pediatrics. She is the director of the Division of Community-Based Research and the director of the Community Wellness Interprofessional Collaborative at the Mailman Center for Child Development. As a pediatric psychologist, she has expertise in health disparities working with ethnically diverse preschool aged children in child care settings. Dr. Natale currently leads four multi-year grants 
examining the effectiveness of health and wellness programing in early childhood populations. For the past 10 years, she has published extensively in the area of health promotion and disease prevention with preschool aged children in underserved areas. She serves on several local and national advisory committees related to early childhood intervention. In 2014, she was honored by the Department of Health's Consortium for a Healthier Miami-Dade as an Outstanding Contributor for the Needs of Children. 